\title{
Intrabeam scattering analysis of measurements at KEK's Accelerator Test Facility damping ring
}

\author{
K. L. F. Bane \\ Stanford Linear Accelerator Center, Stanford University, Stanford, California 94309 \\ H. Hayano, K. Kubo, T. Naito, T. Okugi, and J. Urakawa \\ High Energy Accelerator Research Organization (KEK), 1-1 Oho, Tsukuba, Ibaraki, Japan
}

(Received 4 June 2002; published 27 August 2002)

\begin{abstract}
We derive a simple relation for estimating the relative emittance growth in $x$ and $y$ due to intrabeam scattering (IBS) in electron storage rings. We show that IBS calculations for the Accelerator Test Facility (ATF) damping ring, when using the formalism of Bjorken-Mtingwa, a modified formalism of Piwinski (where $\eta^{2} / \beta$ has been replaced by $\mathcal{H}$ ), or a simple high-energy approximate formula all give results that agree well. Comparing theory, including the effect of potential well bunch lengthening, with a complete set of ATF steady-state beam size versus current measurements we find reasonably good agreement for energy spread and horizontal emittance. The measured vertical emittance, however, is larger than theory in both offset (zero current emittance) and slope (emittance change with current). Almost all the offset error can be accounted for by considering the expected projected vertical emittance due to machine errors rather than the real emittance. This result is consistent with the assumed Coulomb log factor being close to the correct one. The slope error indicates measurement error and/or additional current-dependent physics at the ATF.
\end{abstract}

DOI: 10.1103/PhysRevSTAB.5.084403

PACS numbers: 29.27.Fh, 29.20.Dh, 41.75.Ht

\section{INTRODUCTION}

In future $e^{+} e^{-}$linear colliders, such as the JLC/NLC $[1,2]$, damping rings are needed to generate beams of intense bunches with low emittances. The Accelerator Test Facility (ATF) [3] at KEK is a prototype of such damping rings. One of its main goals, and one that has been achieved, was the demonstration of extremely low vertical emittances [4,5]. At the low ATF emittances, however, it is found that intrabeam scattering (IBS) is a strong effect, and one that needs to be understood. Intrabeam scattering is an effect that depends on the ring lattice - including the errors - and on all dimensions of the beam, including the energy spread. At the ATF all these dimensions can be measured; unique to the ATF is that the beam energy spread, an especially important parameter in IBS theory, can be measured to an accuracy of a few percent. In April 2000 the single bunch energy spread, bunch length, and horizontal and vertical emittances were all measured as functions of current over a short period of time [6,7]. The short period of time was important to ensure that the machine conditions remained unchanged; the bunch length measurement was important since potential well bunch lengthening is significant at the ATF [7]. The question that we attempt to answer here is, are these measurement results in accord with IBS theory?

Intrabeam scattering theory was first developed for accelerators by Piwinski [8], a result that was extended by Martini [9], to give a formulation that we call here the standard Piwinski (P) method [10]; this was followed by the equally detailed Bjorken and Mtingwa (BM) result [11]. Both approaches solve the local, two-particle Coulomb scattering problem for (six-dimensional) Gaussian, uncoupled beams, but the two results appear to be different; of the two, the BM result is thought to be the more general [12]. Other simpler, more approximate formulations developed over the years are ones due to Parzen [13], Le Duff [14], Raubenheimer [15], and Wei [16]. Recent reports on IBS theory include one by Kubo and Oide, who adapt an intermediate result from Bjorken-Mtingwa's paper to find the solution for cases of arbitrary coupling [17], a method that is now used in the optics computer program SAD [18]; and one by Venturini that solves for IBS in the presence of a strong ring impedance [19].

Intrabeam scattering measurements have been performed primarily on hadronic [20-23] and heavy ion machines [24,25], where the effect tends to be more pronounced, though measurement reports on low emittance electron rings can also be found [26,27]. Typical of such reports, however, is that although good agreement may be found in some beam dimension(s), the set of measurements and/or agreement is not complete (e.g., in Ref. [20] growth rates agree reasonably well in the longitudinal and horizontal directions, but completely disagree in the vertical). Note that one advantage of studying IBS using electron machines is that it can be done by measuring steady-state beam sizes. In this report we briefly describe intrabeam scattering formulations, apply and compare them for ATF parameters, and finally compare calculations with the full set of data of April 2000. For more details on the hardware and such measurements at the ATF, the reader is referred to Refs. [4,5].

\section{IBS CALCULATIONS}

We begin by describing the method of calculating the effect of IBS in a storage ring. Let us first assume that there is no $x-y$ coupling. 
Let us consider the IBS growth rates in energy $p$, in the horizontal $x$, and in the vertical $y$ to be defined as

$$
\begin{gathered}
\frac{1}{T_{p}}=\frac{1}{\sigma_{p}} \frac{d \sigma_{p}}{d t}, \quad \frac{1}{T_{x}}=\frac{1}{\epsilon_{x}^{1 / 2}} \frac{d \epsilon_{x}^{1 / 2}}{d t}, \\
\frac{1}{T_{y}}=\frac{1}{\epsilon_{y}^{1 / 2}} \frac{d \epsilon_{y}^{1 / 2}}{d t} .
\end{gathered}
$$

Here $\sigma_{p}$ is the rms (relative) energy spread, $\epsilon_{x}$ the horizontal emittance, and $\epsilon_{y}$ the vertical emittance. In general, the growth rates are given in both $\mathrm{P}$ and $\mathrm{BM}$ theories in the form:

$$
\frac{1}{T_{i}}=\left\langle f_{i}\right\rangle
$$

where subscript $i$ stands for $p, x$, or $y$. The functions $f_{i}$ are integrals that depend on beam parameters, such as energy and phase space density, and lattice properties, including dispersion; the brackets \langle\rangle mean that the quantity is averaged over the ring. In this report we will primarily use the $f_{i}$ of the BM formulation [28].

From the $1 / T_{i}$ we obtain the steady-state properties for machines with radiation damping:

$$
\begin{gathered}
\epsilon_{x}=\frac{\epsilon_{x 0}}{1-\tau_{x} / T_{x}}, \quad \epsilon_{y}=\frac{\epsilon_{y 0}}{1-\tau_{y} / T_{y}}, \\
\sigma_{p}^{2}=\frac{\sigma_{p 0}^{2}}{1-\tau_{p} / T_{p}},
\end{gathered}
$$

where subscript 0 represents the beam property due to synchrotron radiation alone, i.e., in the absence of IBS, and the $\tau_{i}$ are synchrotron radiation damping times. These are three coupled equations since all three IBS rise times depend on $\epsilon_{x}, \epsilon_{y}$, and $\sigma_{p}$.

The way of solving Eqs. (3) that we employ is to convert them into three coupled differential equations, such as is done in, e.g., Ref. [29], and solve for the asymptotic values. For example, the equation for $\epsilon_{y}$ becomes

$$
\frac{d \epsilon_{y}}{d t}=-\frac{2\left(\epsilon_{y}-\epsilon_{y 0}\right)}{\tau_{y}}+\frac{2 \epsilon_{y}}{T_{y}},
$$

and there are corresponding equations for $\epsilon_{x}$ and $\sigma_{p}^{2}$.

Before solving these equations one needs to know the source of the vertical emittance at zero current. We consider three possible sources: (i) vertical dispersion due to vertical orbit errors, (ii) (weak) $x-y$ coupling due to such things as rolled quads, etc., and (iii) a combination of the two. If the vertical emittance at zero current is due mainly to vertical dispersion, then [15]

$$
\epsilon_{y 0} \approx J_{\epsilon}\left\langle\mathcal{H}_{y}\right\rangle \sigma_{p 0}^{2},
$$

with $J_{\epsilon}$ the energy damping partition number and $\mathcal{H}=$ $\left[\eta^{2}+\left(\beta \eta^{\prime}-\frac{1}{2} \beta^{\prime} \eta\right)^{2}\right] / \beta$ the dispersion invariant, with $\eta$ and $\beta$, respectively, the lattice dispersion and beta functions. If $\epsilon_{y 0}$ is mainly due to coupling we drop the $\epsilon_{y}$ differential equation and simply let $\epsilon_{y}=\kappa \epsilon_{x}$, with $\kappa$ the coupling factor. In case (iii) we approximate the solution by replacing the parameter $\epsilon_{y 0}$ in Eq. (4) by the quantity $\left[\kappa \epsilon_{x}\left(1-\tau_{y} / T_{y}\right)+\epsilon_{y 0 d}\right]$, where $\epsilon_{y 0 d}$ is the part of $\epsilon_{y 0}$ due to dispersion only. Note that the practice-sometimes found in the literature - of solving IBS equations assuming no vertical errors, which tends to result in near 0 or even negative vertical emittance growth, may describe a state that is unrealistic and unachievable. Note also that in case (i) once the vertical orbit - and therefore $\left\langle\mathcal{H}_{y}\right\rangle$ - is set, $\epsilon_{y 0}$ is no longer a free parameter.

In addition, note the following.

(1) A fourth equation in our system, the relation between bunch length $\sigma_{s}$ and $\sigma_{p}$, is also implied; generally this is taken to be the nominal (zero current) relation. In the ATF strong potential well bunch lengthening, though no microwave instability, is found at the highest single bunch currents [7]. In our comparisons with ATF measurements we approximate this effect by adding a multiplicative factor $f_{\mathrm{pw}}(I)$ ( $I$ is current), obtained from measurements, to the equation relating $\sigma_{s}$ to $\sigma_{p}$. (Note that potential well bunch lengthening also changes the longitudinal bunch shape, a less important effect that we will ignore.)

(2) The BM results include a so-called Coulomb log factor, $\ln \left(b_{\max } / b_{\min }\right)$, with $b_{\max }, b_{\min }$ maximum, minimum impact parameters, quantities which are not well defined. For round beams it seems that $b_{\max }$ should be taken as the beam size [30]. For bi-Gaussian beams it is not clear what the right choice is. Normally $b_{\max }$ is taken to be the vertical beams size, though the choice of the horizontal beam size can also be found in the literature [31]. Fortunately, the result is not very sensitive to the exact choice. We take $b_{\max }=\sigma_{y} ; b_{\min }=r_{0} c^{2} /\left\langle v_{x}^{2}\right\rangle=r_{0} \beta_{x} /\left(\gamma^{2} \epsilon_{x}\right)$, with $r_{0}$ the classical electron radius $\left(=2.82 \times 10^{-15} \mathrm{~m}\right), v_{x}$ the transverse velocity in the rest frame, and $\gamma$ the Lorentz energy factor. For the ATF, the Coulomb $\log ,(\log )=16.0$.

(3) The IBS bunch distributions are not Gaussian, and tail particles can be overemphasized in these solutions. We are interested in core sizes, which we estimate by eliminating interactions with collision rates less than the synchrotron radiation damping rate [32]. We can approximate this in the Coulomb log term by letting $\pi b_{\min }^{2}\left\langle\left|v_{x}\right|\right\rangle\langle n\rangle$ equal the synchrotron damping rate in the rest frame, with $n$ the particle density in the rest frame [17]; or $b_{\min }=$ $\sqrt{4 \pi \sigma_{x} \sigma_{y} \sigma_{z} \gamma /[N c \tau]}\left(\beta_{x} / \epsilon_{x}\right)^{1 / 4}$, with $N$ the bunch population. For the ATF with this cut, $(\log )=10.0$.

\section{A. High-energy approximation}

For both the $\mathrm{P}$ and the BM methods solving for the IBS growth rates is time consuming, involving, at each iteration step, a numerical integration at every lattice element. A quicker-to-calculate, high-energy approximation, one valid in normal storage ring lattices, can be derived from the BM formalism [33]: 


$$
\begin{aligned}
\frac{1}{T_{p}} & \approx \frac{r_{0}^{2} c N(\log )}{16 \gamma^{3} \epsilon_{x}^{3 / 4} \epsilon_{y}^{3 / 4} \sigma_{s} \sigma_{p}^{3}}\left\langle\sigma_{H} g(a / b)\left(\beta_{x} \beta_{y}\right)^{-1 / 4}\right\rangle \\
\frac{1}{T_{x, y}} & \approx \frac{\sigma_{p}^{2}\left\langle\mathcal{H}_{x, y}\right\rangle}{\epsilon_{x, y}} \frac{1}{T_{p}}
\end{aligned}
$$

with

$$
\begin{gathered}
\frac{1}{\sigma_{H}^{2}}=\frac{1}{\sigma_{p}^{2}}+\frac{\mathcal{H}_{x}}{\epsilon_{x}}+\frac{\mathcal{H}_{y}}{\epsilon_{y}}, \\
a=\frac{\sigma_{H}}{\gamma} \sqrt{\frac{\beta_{x}}{\epsilon_{x}}}, \quad b=\frac{\sigma_{H}}{\gamma} \sqrt{\frac{\beta_{y}}{\epsilon_{y}}} .
\end{gathered}
$$

The requirement on high energy is that $a, b \ll 1$; if the beam momentum in the longitudinal plane is much less than in the transverse planes then this requirement is satisfied. For flat beams $a / b$ is less than 1 . In the ATF, for example, when $\epsilon_{y} / \epsilon_{x} \sim 0.01, a \sim 0.01, b \sim 0.1$, and $a / b \sim 0.1$. The function $g$, related to the elliptic integral, can be well approximated by

$$
g(\alpha) \approx \alpha^{(0.021-0.044 \ln \alpha)} \quad(\text { for } 0.01<\alpha<1),
$$

to obtain $g$ for $\alpha>1$, note that $g(\alpha)=g(1 / \alpha)$.

Note that Parzen's high-energy formula is a similar, though more approximate, result to that given here [13]; and Raubenheimer's approximation formula is similar, though less accurate, than the first and identical to the 2nd and 3rd of Eqs. (6) [15]. Note that Eqs. (6) assume that $\epsilon_{y 0}$ is due mainly to vertical dispersion; if it is due mainly to $x-y$ coupling we let $\mathcal{H}_{y}=0$, drop the $1 / T_{y}$ equation, and simply let $\epsilon_{y}=\kappa \epsilon_{x}$. Finally, note that these equations still need to be iterated, as described before, to find the steady-state solutions.

\section{B. Emittance growth theorem}

Following an argument in Ref. [15] we can obtain a relation between the expected vertical and horizontal emittance growth due to IBS in the presence of random vertical dispersion. We assume that the ring vertical dispersion is random, such as might be expected of the residual dispersion after dispersion correction has been performed. We begin by noting that the beam momentum in the longitudinal plane is much less than in the transverse planes. Therefore, IBS will first heat the longitudinal plane; this, in turn, increases the transverse emittances through dispersion [through $\mathcal{H}$, as can be seen in the 2nd and 3rd of Eqs. (6)], like synchrotron radiation (SR) does. One difference between IBS and SR is that IBS increases the emittance everywhere and SR only in bends. We can write

$$
\frac{\boldsymbol{\epsilon}_{y 0}}{\boldsymbol{\epsilon}_{x 0}} \approx \frac{J_{x}\left\langle\mathcal{H}_{y}\right\rangle_{b}}{J_{y}\left\langle\mathcal{H}_{x}\right\rangle_{b}}, \quad \frac{\boldsymbol{\epsilon}_{y}-\boldsymbol{\epsilon}_{y 0}}{\boldsymbol{\epsilon}_{x}-\epsilon_{x 0}} \approx \frac{J_{x}\left\langle\mathcal{H}_{y}\right\rangle}{J_{y}\left\langle\mathcal{H}_{x}\right\rangle},
$$

where $J_{x, y}$ are damping partition numbers, and \langle\rangle$_{b}$ means averaging is done only over the bends. For vertical dispersion due to errors we expect $\left\langle\mathcal{H}_{y}\right\rangle_{b} \approx\left\langle\mathcal{H}_{y}\right\rangle$. Therefore,

$$
r_{\epsilon} \equiv \frac{\left(\epsilon_{y}-\epsilon_{y 0}\right) / \epsilon_{y 0}}{\left(\epsilon_{x}-\epsilon_{x 0}\right) / \epsilon_{x 0}} \approx \frac{\left\langle\mathcal{H}_{x}\right\rangle_{b}}{\left\langle\mathcal{H}_{x}\right\rangle}
$$

which, for the ATF is 1.6. If, however, there is only $x-y$ coupling, $r_{\epsilon}=1$; if there is both vertical dispersion and coupling, $r_{\epsilon}$ will be between $\left\langle\mathcal{H}_{x}\right\rangle_{b} /\left\langle\mathcal{H}_{x}\right\rangle$ and 1.

\section{Numerical comparison}

Let us compare the results of the methods P, BM, and Eqs. (6) when applied to the ATF beam parameters and lattice, with vertical dispersion and no $x-y$ coupling. We take as parameters those given in Table I, and, for this comparison, let $f_{\mathrm{pw}}=1$. In addition we have $J_{\epsilon}=1.4,\left\langle\beta_{x}\right\rangle=3.9 \mathrm{~m},\left\langle\beta_{y}\right\rangle=4.5 \mathrm{~m}$, $\left\langle\eta_{x}\right\rangle=5.2 \mathrm{~cm}$, and $\left\langle\mathcal{H}_{x}\right\rangle=2.9 \mathrm{~mm}$. To generate vertical dispersion we randomly offset magnets by $15 \mu \mathrm{m}$ and then calculate the closed orbit using SAD. For our seed we find that the rms dispersion $\left(\eta_{y}\right)_{\mathrm{rms}}=7.4 \mathrm{~mm}$, $\left\langle\mathcal{H}_{y}\right\rangle=17 \mu \mathrm{m}$, and $\epsilon_{y 0}=6.9 \mathrm{pm}$ [in agreement with Eq. (5)]. For consistency between the methods we here take the cutoff parameter $d=3 \sigma_{y}$ in $\mathrm{P}$ to correspond to $(\log )=\ln \left[d \sigma_{H}^{2} /\left(4 r_{0} a^{2}\right)\right]=16$ in BM.

Performing the calculations, but first comparing the standard Piwinski and BM methods, we find that the growth rates in $p$ and $x$ agree well; the vertical rate, however, does not. In Fig. 1 we display the local IBS growth rate in $y$ over half the ring (the periodicity is 2), as obtained by the two methods, and see that the P result, on average, is $25 \%$ low. Studying the two methods we note that a conspicuous difference between them is their dependence on dispersion: for $\mathrm{P}$ the $f_{i}$ depend on it only through $\eta^{2} / \beta$; for BM, through $\phi=\left(\eta^{\prime}-\frac{1}{2} \beta^{\prime} \eta / \beta\right)$ and through $\mathcal{H}$. Let us replace $\eta^{2} / \beta$ in $\mathrm{P}$ with $\mathcal{H}$ to create a method that we call the modified Piwinski result. In Ref. [33] it is shown that, in a normal storage ring lattice, at high energies, the results of this method become equal to those of BM.

Comparing with this method we find that, indeed, the three growth rates now agree reasonably well with the BM result. Figure 2 displays the three local growth rates as obtained by the modified $\mathrm{P}$ and BM methods. The $1 / T_{i}$, the average values of these functions, are given in Table II. We note that the $\mathrm{P}$ results are all slightly low, by $4.5 \%$. The

TABLE I. Typical ATF parameters in single bunch mode.

\begin{tabular}{lccc}
\hline \hline Circumference & $C$ & 138 & $\mathrm{~m}$ \\
Energy & $E$ & 1.28 & $\mathrm{GeV}$ \\
Current & $I$ & 3.1 & $\mathrm{~mA}$ \\
Nominal energy spread & $\sigma_{p 0}$ & 5.44 & $10^{-4}$ \\
Nominal horizontal emittance & $\epsilon_{x 0}$ & 1.05 & $\mathrm{~nm}$ \\
Nominal bunch length & $\sigma_{s 0}$ & $5.06^{\mathrm{a}}$ & $\mathrm{mm}$ \\
Longitudinal damping time & $\tau_{p}$ & 20.9 & $\mathrm{~ms}$ \\
Horizontal damping time & $\tau_{x}$ & 18.2 & $\mathrm{~ms}$ \\
Vertical damping time & $\tau_{y}$ & 29.2 & $\mathrm{~ms}$ \\
\hline \hline
\end{tabular}

${ }^{\mathrm{a} A t}$ rf voltage $300 \mathrm{kV}$. 


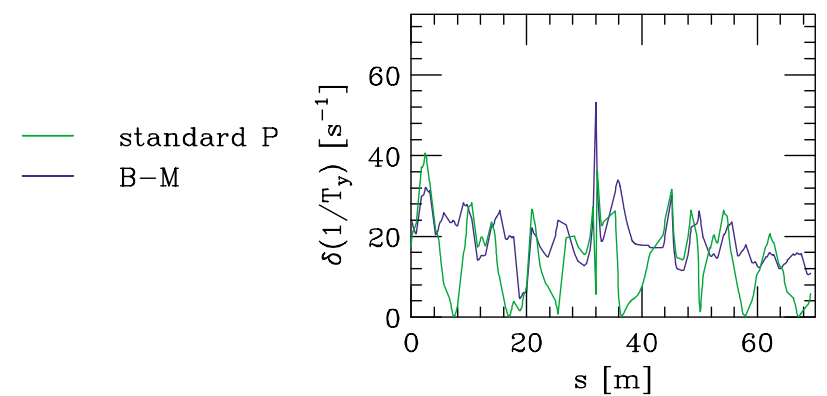

FIG. 1. (Color) Vertical steady-state (local) growth rate over $1 / 2$ the ATF for an example with vertical dispersion due to random errors. Given are results due to standard Piwinski (green curve) and Bjorken-Mtingwa (blue curve).

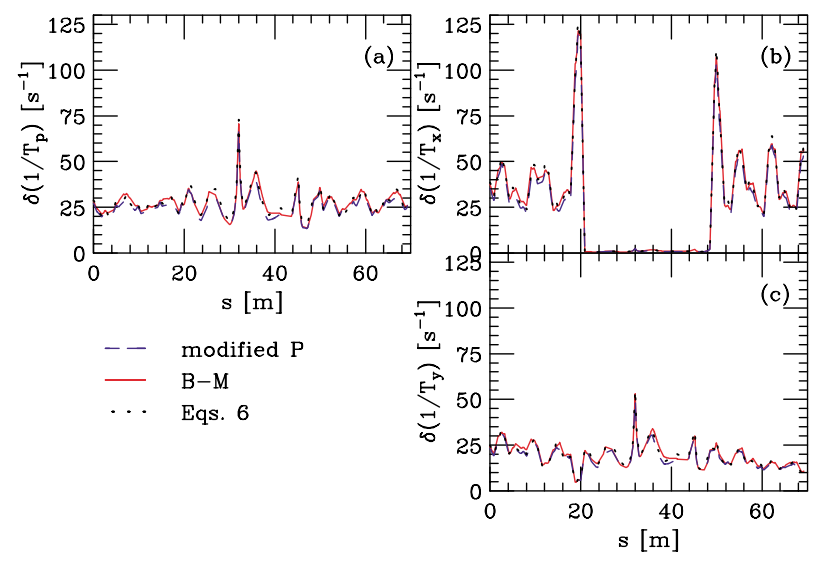

FIG. 2. (Color) Steady-state (local) growth rates over $1 / 2$ the ATF for an example with vertical dispersion due to random errors. Given are results due to modified Piwinski, BjorkenMtingwa, and Eqs. (6).

TABLE II. Steady-state IBS growth rates for an example including vertical dispersion due to random errors.

\begin{tabular}{lccc}
\hline \hline \multicolumn{1}{c}{ Method } & $1 / T_{p}\left(\mathrm{~s}^{-1}\right)$ & $1 / T_{x}\left(\mathrm{~s}^{-1}\right)$ & $1 / T_{y}\left(\mathrm{~s}^{-1}\right)$ \\
\hline Modified Piwinski & 25.9 & 24.7 & 18.5 \\
Bjorken-Mtingwa & 27.0 & 26.0 & 19.4 \\
Eqs. (6) & 27.4 & 26.0 & 19.4 \\
\hline \hline
\end{tabular}

BM method gives $\sigma_{p} / \sigma_{p 0}=1.52, \epsilon_{x} / \epsilon_{x 0}=1.90$, and $\epsilon_{y} / \epsilon_{y 0}=2.30$. Note that for this error seed the emittance growth ratio of Eq. (11) is $r_{\epsilon}=1.44$, close to the 1.6 expected for the ATF lattice.

Repeating the calculation using Eqs. (6) we find that the computing time is greatly reduced, and the growth rates agree quite well with the BM results (see Table II). The dots in Fig. 2 give the local rates corresponding to Eqs. (6), and we see that even these agree quite well.

\section{Comparison with SAD results}

The optics program SAD basically follows the BM formalism, but it does it in a form that treats the three beam

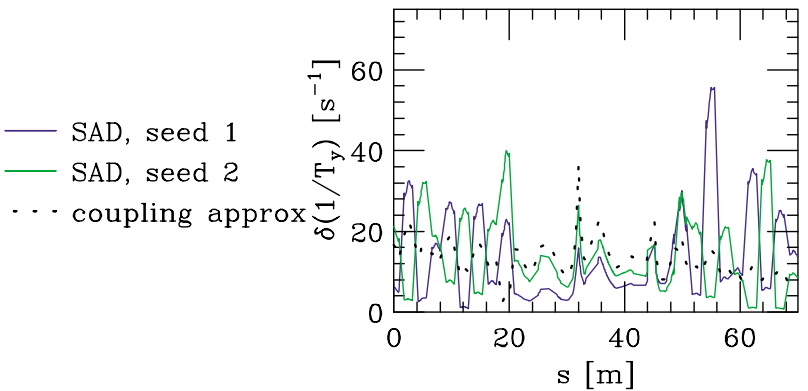

FIG. 3. (Color) Vertical steady-state (local) growth rate over $1 / 2$ the ATF for an example with vertical dispersion and $x-y$ coupling due to random errors. Given are results obtained by SAD (for 2 seeds; solid curves) and by the coupling approximation used here (dots).

TABLE III. Steady-state IBS growth rates for an example including vertical dispersion and $x-y$ coupling due to random errors.

\begin{tabular}{lccc}
\hline \hline \multicolumn{1}{c}{ Method } & $1 / T_{p}\left(\mathrm{~s}^{-1}\right)$ & $1 / T_{x}\left(\mathrm{~s}^{-1}\right)$ & $1 / T_{y}\left(\mathrm{~s}^{-1}\right)$ \\
\hline SAD, seed 1 & 22.5 & 19.6 & 13.1 \\
SAD, seed 2 & 22.3 & 19.6 & 13.5 \\
Our approx. calculation & 22.9 & 21.0 & 12.9 \\
\hline \hline
\end{tabular}

directions on equal footing. The final results are given in terms of the normal modes of the system and not the beta and dispersion functions of the uncoupled system (as in our approximation). For vertical dispersion dominated problems there is no difference in the result. In coupling dominated problems there will be a difference in growth rates that, in the case of small $x-y$ coupling due to errors, we expect, on average, to be small.

We consider the ATF lattice with random magnet offsets and rotations. Other machine parameters are the same as before; again $I=3.1 \mathrm{~mA}$. For this lattice $\left(\eta_{y}\right)_{\mathrm{rms}}=$ $7.4 \mathrm{~mm}$ and $\epsilon_{y 0} / \epsilon_{x 0}=1 \%$. For this problem we solve IBS using SAD (for two different seeds), and also our approximate method where we include vertical dispersion (as before) and a global coupling parameter $\kappa=0.34 \%$. We take $(\log )=9.1$. Comparing steady-state local growth rates, we find good agreement in $p$ and $x$ for all three calculations. In $y$, however, there is a significant variation (see Fig. 3). The growth rates, the average values of these functions, however, agree well (see Table III). Note that the steady-state relative growths in $\left(\sigma_{p}, \epsilon_{x}, \epsilon_{y}\right)$ are $(1.38,1.56,1.64)$ for SAD, and $(1.38,1.62,1.61)$ for our approximate calculation.

\section{COMPARISON WITH MEASUREMENT}

\section{A. Measurements}

At the ATF the energy spread and all beam sizes can be measured. Unique at the ATF is that the energy spread, a particularly important parameter in IBS theory, can be obtained to a few percent accuracy. In this measurement 
the beam is extracted and its size measured on a screen in a highly dispersive region. The bunch length is determined with a streak camera in the ring.

The emittances can be measured using three methods: wire monitors in the extraction line, a laser wire in the ring, and an interferometer in the ring. Unfortunately, for $\epsilon_{y}$ all three methods have their difficulties. The wire measurement is very sensitive to fluctuations in the extraction kicker field, to any nonlinear component of the magnetic field in the extraction line, and to residual dispersion in the extraction line; whenever the vertical-horizontal emittance ratio is small, all of these effects will result in an artificially large measured vertical emittance. The laser wire and interferometer measurements are sensitive to beam oscillations in the ring. The laser wire measurement, being time consuming (taking $\sim 1 \mathrm{~h}$ per measurement), is also sensitive to slow drifts in machine and beam properties. As for the interferometer, defects in the mirrors and mechanical vibrations of the monitors are also possible error sources. Again, the result will tend to be an artificially large measured vertical emittance. For more details, see Refs. [4,5].

Because of the effects of IBS the energy spread measurement (which is quick and easy to perform) has become a useful technique for monitoring changes in beam size. Thus, evidence that we are truly seeing IBS at the ATF include (1) when moving onto the coupling resonance, the normally large energy spread growth with current becomes negligibly small; (2) if we decrease the vertical emittance using dispersion correction, the energy spread increases.

\section{B. Comparison with theory}

In Fig. 4, as an example, we present the time development, after injection, of energy spread for three different

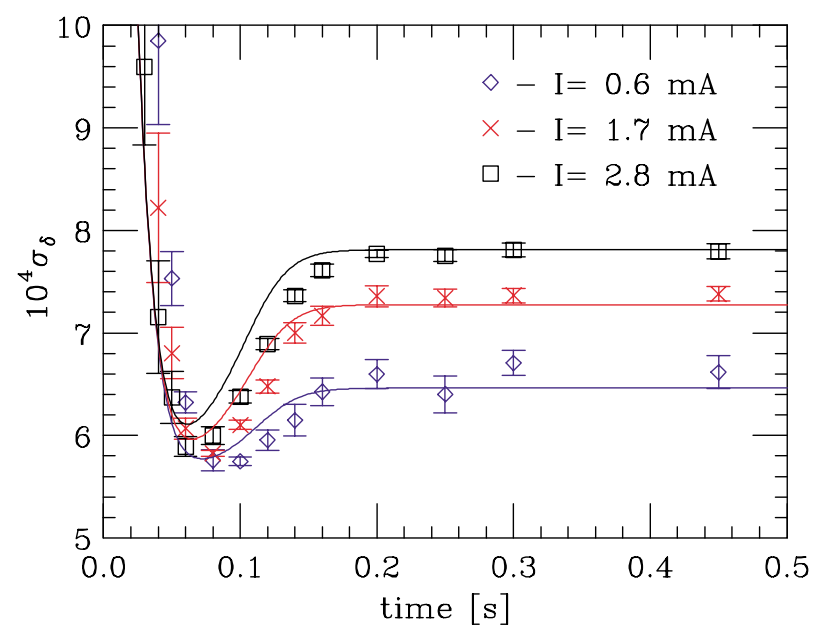

FIG. 4. (Color) Measured energy spread as function of time after injection, for three different currents (the plotting symbols). The curves give BM simulations assuming an $x-y$ coupling of 0.006 and no potential well distortion. This plot is reproduced from Ref. [4]. beam currents (the plotting symbols). The measurement was performed by continually injecting beam into the ATF, while varying the extraction timing. If we take the BM formalism, with $f_{\mathrm{pw}}=1$, and with $x-y$ coupling 0.006 , and solve the differential equations for energy spread and beam sizes, we obtain the curves in the figure (if we include potential well distortion the fitted coupling becomes 0.0045). The short time ( $\leqslant 0.05 \mathrm{~s}$ ) behavior does not agree with the data, since the beam in reality enters the ring badly mismatched (a region which would be difficult to simulate); in the longer time range, however, after $\gtrsim 3 \tau_{p}$, the agreement becomes quite good. The minimum in the curves can be explained as follows: Initially the energy spread and beam sizes reduce due to synchrotron radiation; when the beam volume becomes smaller than a certain amount, the energy spread begins to increase due to IBS. This result indicates reasonably good agreement between measurement and theory.

To compare with theory absolutely, however, we need to measure all beam properties with the machine in the same condition. Such a complete series of measurements was performed on the stored beam at the ATF over a short period of time in April 2000. The rf voltage was $V_{c}=300 \mathrm{kV}$. The energy spread and bunch length versus current measurements are shown in Fig. 5. The curves in the plots are fits that give the expected zero current result. Emittances were measured on the wire monitors in the extraction line (the symbols in Figs. $6 \mathrm{~b}$ and $6 \mathrm{c}$; note that the symbols in Fig. 6a reproduce the fits to the data of Fig. 5). We see large growth also in the emittances. Unfortunately, we have no error bars for the emittance measurements, though we expect the random component of errors in $y$ to be $5 \%-10 \%$ and less in $x$. Note that $\epsilon_{y 0}$ appears to be about $1.0 \%-1.2 \%$ of $\epsilon_{x 0}$.

Let us compare BM calculations with the data. Here we take $f_{\mathrm{pw}}$ as given by the measurements and take as Coulomb log our best estimate, $(\log )=10$. Note that in the machine the residual dispersion is typically $\left(\eta_{y}\right)_{\mathrm{rms}} \sim$ $3 \mathrm{~mm}$. To set our one free parameter, $\boldsymbol{\epsilon}_{y 0}$, we adjust it until at high current $\sigma_{p}$ agrees with the measurement. In Fig. 6 we give examples.

(1) Vertical dispersion only, with $\left(\eta_{y}\right)_{\mathrm{rms}}=5.6 \mathrm{~mm}$ and $\epsilon_{y 0}=4.0 \mathrm{pm}$ (solid line).

(2) Coupling dominated with $\kappa=0.33 \%$ (dashed line).

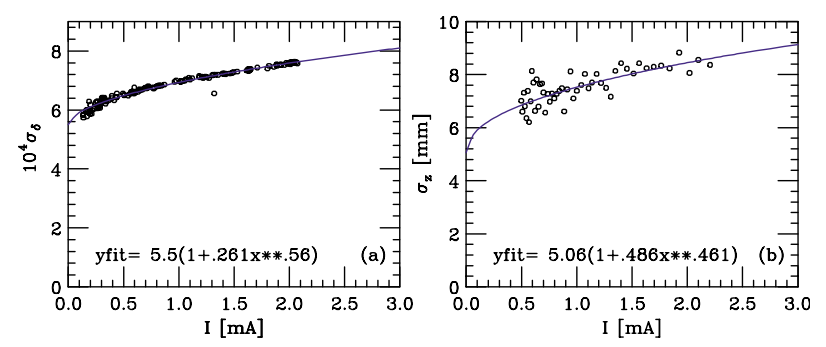

FIG. 5. (Color) Measurements of steady-state energy spread (a) and bunch length (b), with $V_{c}=300 \mathrm{kV}$. 

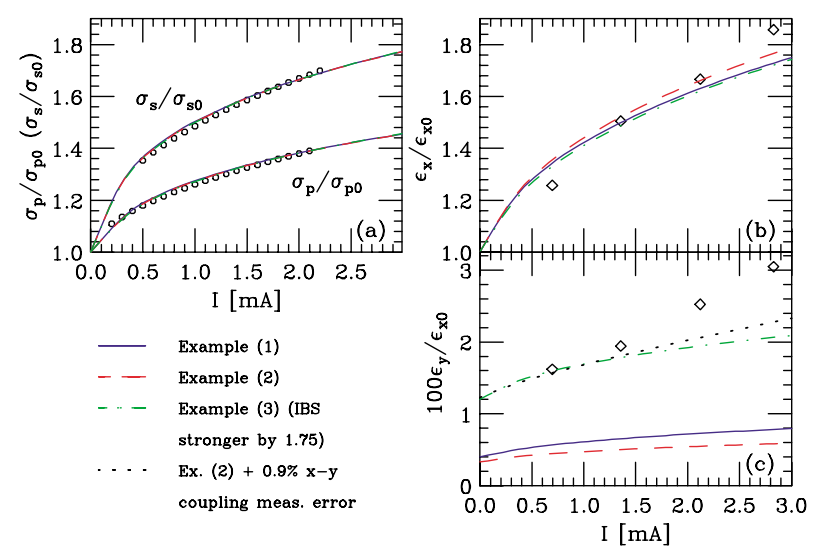

FIG. 6. (Color) ATF measurement data (symbols) and IBS theory fits (the curves). The symbols in (a) give the smooth curve fits to the measured data of Fig. 5.

(3) Coupling dominated with $\kappa=1.2 \%$, with the Coulomb log artificially increased by a factor 1.75 (dot-dashed line).

(4) Same as (2) but assuming $\epsilon_{y}$ measurement error, i.e., adding $0.9 \%$ of the measured (and splined) $\epsilon_{x}$ to the calculated $\epsilon_{y}$ (dotted line).

We see that $\sigma_{p}(I)$ agrees well with the measurements for all cases, and $\epsilon_{x}(I)$ agrees reasonably well. For examples (1) and (2), however, $\epsilon_{y 0}$ is significantly lower than the measurements seem to indicate, and the growth with current is also less. To obtain reasonable agreement for $\epsilon_{y 0}$ we need to assume that either IBS is $\sim 75 \%$ stronger (in growth rates) than theory predicts, or there is significant measurement error, equivalent to $\sim 1 \% x$ emittance coupling into the $y$ measurement. Yet even with such assumptions the $\epsilon_{y}(I)$ dependence does not agree.

What does the emittance growth theorem of Sec. II B say about these results? It appears that $\epsilon_{x}$ grows by $\sim 85 \%$ by $I=3 \mathrm{~mA} ; \epsilon_{y}$ begins at about $1.0 \%-1.2 \%$ of $\epsilon_{x 0}$, and then grows to about $3 \%$ of $\epsilon_{x 0}$. Therefore, the relative emittance growth ratio is $r_{\epsilon} \sim 2.1-2.4$, much larger than the expected result if we are coupling dominated (1.0); and still significantly larger than the expected result if we are dispersion dominated (1.6), a case that is anyway unlikely since it requires an implausibly large $\left(\eta_{y}\right)_{\mathrm{rms}} \approx 9 \mathrm{~mm}$. Thus, the emittance growth theorem indicates that $\epsilon_{y}(I)$ as measured is not in agreement with IBS theory.

\section{Detailed simulation}

To try to understand this discrepancy, we performed detailed SAD simulations with the expected errors. The simulations included orbit errors, including in the sextupoles, and quad and sextupole random rotations (with an rms of $20 \mathrm{mr}$ ). The simulations also included orbit, dispersion, and coupling correction.

At any current the rf voltage was adjusted so that, on average (when averaging the results of many seeds), $f_{\mathrm{pw}}$ was in agreement with measurement (Fig. 6a). Also, our original estimate of the errors was slightly scaled so that, on average, $\sigma_{p}$ at $I=2.8 \mathrm{~mA}$ also agreed with measurement. Then, out of 500 seeds, we picked the 100 cases for which $\sigma_{p}(2.8 \mathrm{~mA})$ agreed with measurement to within $\pm 1.5 \%$. For all these cases, $\sigma_{p}, \sigma_{z}$, and $\epsilon_{x}$ agreed very well (to a few percent) with the solid curves of Figs. $6 \mathrm{a}$ and $6 b$.

For the calculated vertical emittance we found that (i) $\epsilon_{y 0} / \epsilon_{x 0}=(0.5 \pm 0.05) \%$ and, at $I=2.8 \mathrm{~mA}, \epsilon_{y} / \epsilon_{x 0}=$ $(0.8 \pm 0.08) \%$ (very similar to the solid curve of Fig. $6 \mathrm{c}$ ); (ii) when looking at the projected emittance $\tilde{\epsilon}_{y}$, however, we find that $\tilde{\epsilon}_{y 0} / \epsilon_{x 0}=(0.8 \pm 0.2) \%$, and at $I=2.8 \mathrm{~mA}$, $\tilde{\epsilon}_{y} / \epsilon_{x 0}=(1.3 \pm 0.3) \%$. It is the projected emittance that presumably corresponds to the measurements. Since $\epsilon_{y 0}$, according to measurement, appears to be $1-1.2 \%$ of $\epsilon_{x 0}$ we see that there is reasonably good agreement between this and the calculated value of $\tilde{\epsilon}_{y 0}$. We should point out, however, that the current dependence of the projected emittance is still in disagreement with measurement.

\section{DISCUSSION}

Our disagreement in $\epsilon_{y}$ between theory and measurement consists of two parts, an offset part $\left(\epsilon_{y 0}\right)$ and a disagreement in slope $\left(d \epsilon_{y} / d I\right)$. Together they indicate that we have error in theory, additional physics at the ATF, and/or error in measurement.

IBS theory is a mature theory, and the relation between longitudinal and transverse growth rates [the $2 \mathrm{nd}$ and $3 \mathrm{rd}$ of Eqs. (6)] is simple and intuitively easy to understand. The main uncertainty in theory may be with the scale factor, particularly in the Coulomb log factor for beams with elliptical cross section. Yet the Coulomb log is rather insensitive to error in the argument. In addition, a scale factor error can affect only the offset part of the disagreement. We have seen that most of the offset disagreement can be accounted for by the projected emittance, in the presence of the expected errors, being larger than the real emittance. In fact, this result is consistent with the assumed Coulomb $\log$ factor being close to the correct one.

The disagreement in $d \epsilon_{y} / d I$ might be explained by the presence of additional current-dependent physics at the ATF. We have seen that $\sigma_{p}(I)$ and $\epsilon_{x}(I)$ can be made to agree reasonably well between theory and measurement; at the same time, however, the measured $\epsilon_{y}(I)$ grows much faster than predicted. One might, therefore, suspect the presence at the ATF of another current-dependent effect, one that increases the projected vertical emittance - though not the real emittance. An example of such an effect is a $y-z$ tilt of the beam induced by closed orbit distortion in the presence of a transverse impedance [15,34]. More study needs to be done in this direction.

As mentioned before, measuring accurately the small vertical emittances at the ATF is difficult, and, therefore, emittance measurement error is likely responsible for much 
of the disagreement found. We noted that a coupling measurement error in the extraction line wire measurements can affect the offset part of the disagreement; the slope disagreement, however, is not easy to explain assuming measurement error alone (for an attempt in this direction, see e.g., Ref. [35]).

Over the time since April 2000 the systematics of the emittance measurements have improved, especially for the laser wire measurement. Newer results seem to suggest that the April 2000 measured vertical emittance may have been too large $[4,5]$. For the near future we urge that the effort to obtain reliable emittance measurements at the ATF be continued. Measurements should be performed, using orbit correction, to study the difference between the projected and real vertical emittances. In addition, experiments to study the possible existence of other currentdependent effects should also be performed. Ultimately, one goal should be to test the accuracy of theoretical IBS growth rates to the $10 \%-20 \%$ level. Note that once we are successful at such benchmarking experiments, we will be able to use the ATF energy spread measurement as a diagnostic for the absolute emittances of the beam.

\section{CONCLUSION}

We began by describing intrabeam scattering calculations for electron storage rings, focusing on machines with small random magnet offset and roll errors. We derived a simple relation for estimating the relative emittance growth in $x$ and $y$ due to IBS in such machines. We have shown that IBS calculations for the ATF damping ring, when using the formalism of Bjorken-Mtingwa, a modified formalism of Piwinski (where $\eta^{2} / \beta$ has been replaced by $\mathcal{H}$ ), or a simple high-energy approximate formula all give results that agree well. By comparing with numerical results from SAD we have demonstrated that weak coupling due to random magnet roll can be approximated by solving the uncoupled problem with the addition of a global coupling parameter.

Comparing the BM calculations, and including the effect of potential well bunch lengthening, with a complete set of ATF steady-state energy spread and beam size versus current measurements we have found reasonably good agreement in energy spread and horizontal emittance. At the same time, however, we find that the measured vertical emittance is larger than theory in both offset (zero current emittance) and slope (emittance change with current). Almost all the offset error can be accounted for by considering the expected projected vertical emittance due to machine errors rather than the real emittance. This result is consistent with the assumed Coulomb log factor being close to the correct one. The slope error indicates measurement error and/or the presence of additional currentdependent physics at the ATF. More study is needed.

\section{ACKNOWLEDGMENTS}

The authors thank students, staff, and collaborators on the ATF project. We thank, in particular, M. Ross and A. Wolski for helpful discussions on IBS. One author (K. L. F. B.) also thanks A. Piwinski for guidance on the topic of IBS. This work was supported by the Department of Energy, Contract No. DE-AC03-76SF00515.

[1] KEK Report No. 97-1, 1997.

[2] The NLC Design Group, Stanford Report No. SLAC 474, 1996.

[3] F. Hinode et al., KEK Internal Report No. 95-4, 1995 (unpublished).

[4] K. Kubo et al., Phys. Rev. Lett. 88, 194801 (2002).

[5] H. Sakai et al., KEK Report No. 2002-5, 2002 (to be published).

[6] J. Urakawa, in Proceedings of the 7th European Particle Accelerator Conference (EPAC2000), Vienna, Austria (Austrian Academy of Sciences Press, Vienna, Austria, 2000), p. 63.

[7] K. Bane, T. Naito, T. Okugi, Q. Qin, and J. Urakawa, SLAC Report No. SLAC-PUB 8846, 2001.

[8] A. Piwinski, Stanford Report No. HEAC 74, 1974.

[9] M. Martini, CERN Report No. CERN-PS 84-9 (AA), 1984.

[10] A. Piwinski, in Handbook of Accelerator Physics and Engineering, edited by A.W. Chao and M. Tigner (World Scientific, Singapore, 1999), p. 125.

[11] J.D. Bjorken and S. K. Mtingwa, Part. Accel. 13, 115 (1983).

[12] A. Piwinski (private communication).

[13] G. Parzen, Nucl. Instrum. Methods Phys. Res., Sect. A 256, 231 (1987).

[14] J. Le Duff, in Proceedings of the CERN Accelerator School: Second Advanced Accelerator Physics Course (CERN, Geneva, 1989).

[15] T. Raubenheimer, Ph.D. thesis, Stanford University (Report No. SLAC-R-387, Sec. 2.3.1, 1991).

[16] J. Wei, in Proceedings of the 1993 Particle Accelerator Conference (PAC 93), Washington, D.C. (IEEE, Piscataway, NJ, 1993), p. 3651.

[17] K. Kubo and K. Oide, Phys. Rev. ST Accel. Beams 4, 124401 (2001).

[18] K. Oide, SAD Users Guide, KEK.

[19] M. Venturini, in Proceedings of the 2001 Particle Accelerator Conference (PAC 2001), Chicago (IEEE, Piscataway, NJ, 2001), p. 2819.

[20] M. Conte and M. Martini, Part. Accel. 17, 1 (1985).

[21] L. Evans and J. Gareyte, IEEE Trans. Nucl. Sci. 32, 2234 (1986).

[22] C. Bhat, L. Spentzouris, and P. Colestock, in Proceedings of the 1999 Particle Accelerator Conference (PAC 1999), New York (IEEE, Piscataway, NJ, 1999), p. 3155.

[23] M. Zorzano and R. Wanzenberg, CERN Report No. CERN-SL 2000-072 AP, HERA, 2000.

[24] Y.-N. Rao and L. Hermansson, in Proceedings of the 7th European Particle Accelerator Conference (EPAC2000), Vienna, Austria (Ref. [6]), p. 1549. 
[25] W. Fischer et al., in Proceedings of the 2001 Particle Accelerator Conference (PAC 2001), Chicago (Ref. [19]), p. 2857.

[26] C. Kim, LBL Report No. LBL 42305, 1998.

[27] C. Steier et al., in Proceedings of the 2001 Particle Accelerator Conference (PAC 2001), Chicago (Ref. [19]), p. 2938.

[28] We believe that the right-hand side of Eq. 4.17 in BM (with $\sigma_{\eta}$ equal to our $\sqrt{2} \sigma_{p}$ ) should be divided by $\sqrt{2}$, in agreement with the recent derivation of Ref. [19]. Also, vertical dispersion, though not originally in BM, can be added in the same manner as horizontal dispersion.
[29] C. H. Kim, in Proceedings of the 1997 IEEE Particle Accelerator Conference (PAC 97), Vancouver (IEEE, Piscataway, NJ, 1997), p. 790.

[30] R. Farouki and E. Salpeter, Astrophys. J. 427, 676 (1994).

[31] J. Wei and G. Parzen, in Proceedings of the 2001 Particle Accelerator Conference (PAC 2001), Chicago (Ref. [19]), p. 42.

[32] T. Raubenheimer, Part. Accel. 45, 111 (1994).

[33] K. Bane, SLAC Report No. SLAC-PUB 9226, 2002.

[34] A. W. Chao and S. Kheifets, IEEE Trans. Nucl. Sci. 30, 2571 (1983).

[35] M. Ross and K. Bane, KEK ATF Report No. 01-04, 2001. 